\title{
Prevalence of cognitive impairment in Chinese: Epidemiology of Dementia in Singapore study
}

\author{
Saima Hilal, ${ }^{1}$ Mohammad Kamran Ikram, ${ }^{2,3,4,5}$ Monica Saini, ${ }^{1}$ Chuen Seng Tan, ${ }^{6}$ \\ Joseree Ann Catindig, ${ }^{7}$ Yan Hong Dong, ${ }^{1}$ Leon Ben Swie Lim, ${ }^{1}$ Eric Y S Ting, ${ }^{8}$ \\ Edward H Koo, ${ }^{9}$ Carol Y L Cheung, ${ }^{2,3}$ Anqi Qiu, ${ }^{10,11,12}$ Tien Yin Wong, ${ }^{2,3}$ \\ Christopher Li-Hsian Chen, ${ }^{1}$ Narayanaswamy Venketasubramanian ${ }^{5}$
}

SH and MKI contributed equally to this work.

For numbered affiliations see end of article.

\section{Correspondence to}

Dr M K Ikram, Department of Ophthalmology, National University Health System, $1 \mathrm{E}$ Kent Ridge Road, NUHS Tower Block, Level 7, Singapore 119228, Singapore: kamran_ikram@nuhs.edu.sg

Received 2 September 2012 Revised 10 November 2012 Accepted 11 January 2013 Published Online First 5 February 2013
To cite: Hilal S, Ikram MK Saini M, et al. J Neurol Neurosurg Psychiatry 2013;84:686-692.

\section{ABSTRACT \\ Objective To study the prevalence of and associated factors for cognitive impairment and dementia in community dwelling Chinese from Singapore.}

Methods This study includes Chinese subjects from the Epidemiology of Dementia in Singapore (EDIS) study, aged $\geq 60$ years, who underwent comprehensive examinations, including cognitive screening with the locally validated Abbreviated Mental Test and Progressive Forgetfulness Questionnaire. Screen positive participants subsequently underwent extensive neuropsychological testing and cerebral MRI. Cognitive impairment no dementia (CIND) and dementia were diagnosed according to internationally accepted criteria. The prevalence of cognitive impairment and dementia were computed per 5 year age categories and gender. To examine the relationship between baseline associated factors and cognitive impairment, we used logistic regression models to compute odd ratios with $95 \% \mathrm{Cl}$. Results 1538 Chinese subjects, aged $\geq 60$ years, underwent cognitive screening: $171(15.2 \%)$ were diagnosed with any cognitive impairment, of whom 84 were CIND mild, 80 CIND moderate and seven had dementia. The overall age adjusted prevalence of CIND mild was 7.2\%; CIND moderate/dementia was $7.9 \%$. The prevalence increased with age, from $5.9 \%$ in those aged $60-64$ years to $31.3 \%$ in those aged $75-79$ years and $44.1 \%$ in those aged $\geq 80$ years. Multivariate analysis revealed age, diabetes and hyperlipidaemia to be independently associated with cognitive impairment. Conclusions In present study, the overall prevalence of cognitive impairment and dementia in Chinese was $15.2 \%$, which is in the same range as the prevalence reported in Caucasian and other Asian populations.

\section{INTRODUCTION}

The population of Asia in 2009 was estimated at 4 billion, $59 \%$ of a global total of 6.8 billion. ${ }^{1}$ It is expected that the proportion of older persons aged $\geq 60$ years among the total Asian population will rise from $10 \%$ in 2010 to $24 \%$ in 2050 , and also that the absolute number of elderly will dramatically increase from 414 million to 1.2 billion. $^{2}$ As a result of this rapid demographic aging, the burden from common age related brain diseases, such as dementia, is also expected to rise. The prevalence of dementia in Asia has previously been found to be lower than in western populations, but recent studies suggest that age specific prevalence rates may be similar. ${ }^{3}$

With respect to the Chinese population, prevalence estimates of dementia from China and Singapore varied from $1.2 \%$ to $7.5 \%$ in those aged $>50$ years. ${ }^{4}{ }^{5}$ Moreover, in the past few decades the focus has shifted towards the preclinical stages of dementia, such as cognitive impairment no dementia (CIND). Previous studies in Caucasian populations reported a prevalence ranging from $14.9 \%$ to $22.2 \%$, and in Asians, including Chinese, around 7.7-22.2\%. ${ }^{6} 7$ However, comparison between studies is hampered due to differences in case ascertainment, demographic factors and lack of extensive neuropsychological testing.

In view of the limited knowledge of the prevalence of cognitive impairment among Asians, we initiated a new population based study in Singapore to investigate the prevalence and associated factors of cognitive impairment in a Chinese population from Singapore.

\section{METHODS}

Study design and study population

The Epidemiology of Dementia in Singapore (EDIS) study comprised subjects from the ongoing population based community dwelling study of Chinese aged 40-85 years who participated in the Singapore Chinese Eye Study (SCES). Of the 4605 eligible persons, a total of 3353 participated (participation rate $72.8 \%$ ). Ethics approval for the EDIS study was obtained from the Singapore Eye Research Institute and the National Healthcare Group Domain Specific Review Board. The study was conducted in accordance with the Declaration of Helsinki. Written informed consent was obtained, in the preferred language of participants, by bilingual study coordinators prior to recruitment into the study.

Participants in the SCES study were randomly selected from the community and were invited to the Singapore Eye Research Institute for interview and clinical assessments, as described previously. ${ }^{8} 9$ Information on participants was collected by means of a questionnaire, physical examination and laboratory based tests. The questionnaire included data on demographics, lifestyle factors, personal and family health history, and medication use. Physical examination included anthropometry, blood pressure, pulse rate measurement and 
extensive eye examination, including digital fundal photography. Laboratory examinations included serum creatinine, serum lipids, plasma glucose, glycosylated haemoglobin and urine for albumin and creatinine. Blood samples were stored for future biomarker and genetic analysis.

In the first phase of the EDIS study, SCES participants who were $\geq 60$ years old $(n=1538)$ underwent cognitive screening using the Abbreviated Mental Test and a self-report of progressive forgetfulness, both of which have been previously validated in Singapore. ${ }^{10-14}$ Screen positives were defined as Abbreviated Mental Test $\leq 6$ among those with up to 6 years of formal education or $\leq 8$ among those with more than 6 years of formal education, or if the subject or caregiver said yes to progressive forgetfulness. Those who were screen negative were considered to be cognitively normal. Screen positive subjects were invited to participate in the second phase of the EDIS study. Participants who declined the initial invitation were contacted again at a later time. Those who declined at the first attempt were mailed study brochures, and offered free transportation and pick up services. A person is termed 'uncontactable' if he/ she fails to respond after six attempts.

\section{Examination procedures}

During the second phase, participants underwent extensive clinical and neuropsychological evaluation, along with laboratory tests and neuroimaging, as detailed below (figure 1).

\section{Questionnaire}

A detailed questionnaire was administered by the interviewer to collect relevant demographic and medical information. Data collected included age, gender, education, marital status, occupation, ability to live independently, handedness, previous head trauma, smoking, alcohol consumption and family history of dementia. Previous medical history including stroke, cardiovascular diseases, hypertension, hyperlipidaemia, diabetes mellitus, vitamin B 12 deficiency, thyroid disease, urinary and bowel incontinence, Parkinson's disease, depressive symptoms and psychiatric illnesses were noted, and subsequently verified by medical records. The Instrumental Activities of Daily Living and
Barthel Activities of Daily Living indices were assessed for functional status. ${ }^{15} 16$

Physical examination and clinical assessment

Clinical assessment included height, weight, blood pressure, pulse rate, ankle brachial index, modified versions of the National Institutes of Health Stroke Scale, Hachinski Ischaemic Scale and frontal release signs. Clinical history, examinations and Clinical Dementia Rating Scale evaluations were performed by clinicians.

\section{Blood tests}

A total of $20 \mathrm{ml}$ of blood were drawn in the fasting state. All blood samples were sent to the National University Hospital Laboratory for measurements on the same day. Blood tests included: full blood count, glucose, lipids, creatinine, alanine transaminase, aspartate transaminase, calcium, albumin, thyroid function, vitamin B12, folate, syphilis screen, homocysteine and high sensitivity $\mathrm{C}$ reactive protein.

\section{Neuroimaging}

MRI scans were performed on a $3 \mathrm{~T}$ Siemens Magnetom Trio Tim scanner, using a 32 channel head coil, at the Clinical Imaging Research Centre of the National University of Singapore. A number of standardised and advanced MRI brain sequences were performed to allow morphological, microstructural and functional assessments. Scanning time was approximately $60 \mathrm{~min}$. Subjects with claustrophobia, contraindications for MRI or those who are unable to tolerate the procedure underwent a non-contrast enhanced CT scan, which was performed in axial slices at $5 \mathrm{~mm}$ intervals rostrally from the orbitomeatal line. Scanning time was approximately $3 \mathrm{~min}$. There were 17 subjects in total who had contraindications or refused to undergo MRI, and instead underwent CT scan.

\section{Cognitive impairment and dementia assessment}

Neuropsychological test battery

Trained research psychologists administered brief cognitive screening tests, the Mini-Mental State Examination and the

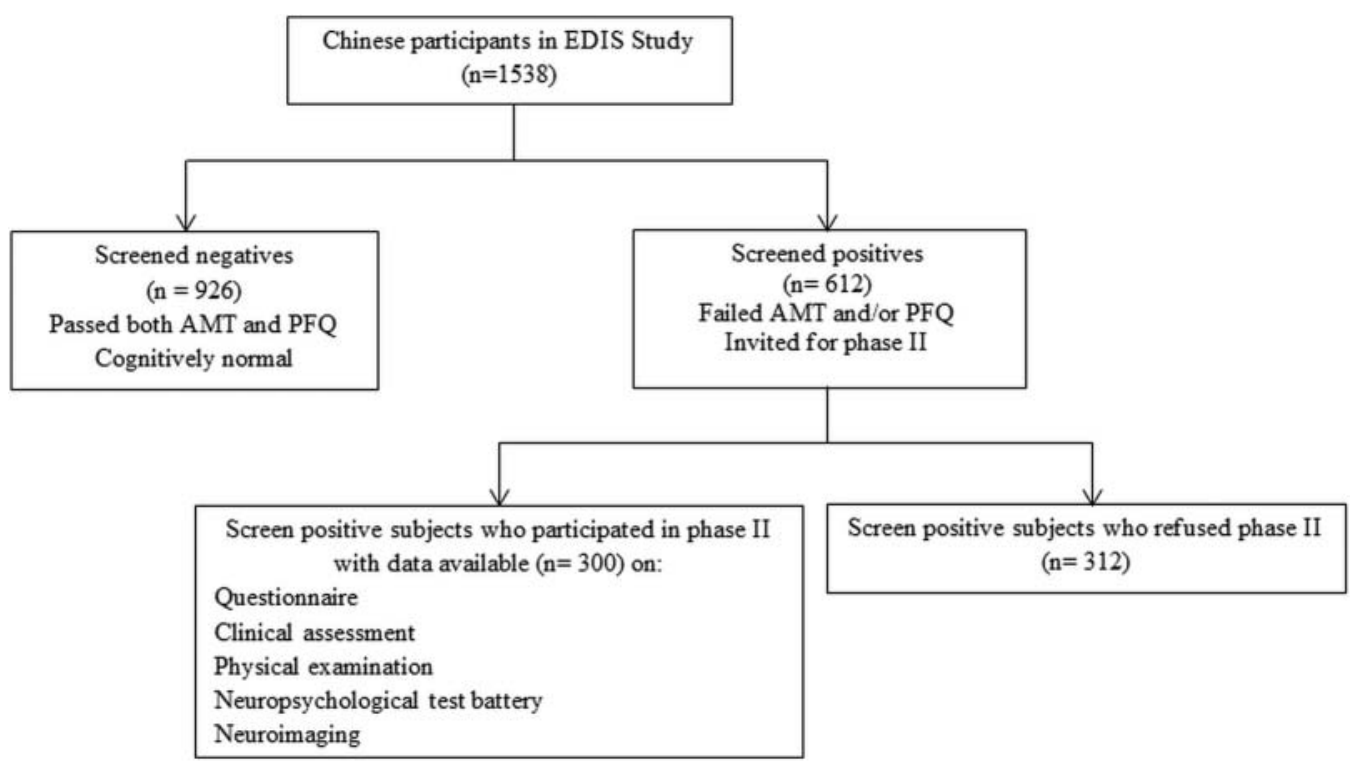

Figure 1 Flowchart of participants in the Epidemiology of Dementia in Singapore Study (EDIS). AMT, Abbreviated Mental Test; PFQ, Progressive Forgetfulness Questionnaire. 
Montreal Cognitive Assessment, the Informant Questionnaire on Cognitive Decline in the Elderly and a formal neuropsychological battery locally validated for Singaporean elderly. ${ }^{17}$ This battery assesses seven domains, five of which are non-memory domains.

The non-memory domains tested were:

- Executive function: Frontal Assessment Battery ${ }^{18}$ and Maze Task ${ }^{19}$

- Attention: Digit Span, Visual Memory Span ${ }^{20}$ and Auditory Detection ${ }^{21}$

- Language: Boston Naming Test ${ }^{22}$ and Verbal Fluency ${ }^{23}$

- Visuomotor speed: Symbol Digit Modality Test ${ }^{24}$ and Digit Cancellation $^{25}$

- Visuoconstruction: Weschler Memory Scale-Revised Visual Reproduction Copy task, ${ }^{20}$ Clock Drawing ${ }^{26}$ and Weschler Adult Intelligence Scale-Revised subtest of Block Design. ${ }^{27}$

The memory domains tested were:

- Verbal memory: Word List Recall ${ }^{11}$ and Story Recall

- Visual memory: Picture Recall and Weschler Memory Scale-Revised Visual Reproduction. ${ }^{20}$

The assessment was administered according to the subject's habitual language and was completed in approximately $1 \mathrm{~h}$. In addition, we also performed the Geriatric Depression Scale, ${ }^{28}$ and the 12 item Neuropsychiatric Inventory ${ }^{29}$ was administered to assess self-reported depressive symptoms and informant reports on the presence, frequency, severity and resulting caregiver distress associated with neuropsychiatric symptoms.

\section{Diagnosis of cognitive impairment and dementia}

Diagnoses of cognitive impairment and dementia were made at weekly consensus meetings attended by study clinicians, neuropsychologists, clinical research fellows, research coordinators and research assistants. Clinical features, blood investigations, psychometrics and neuroimages were reviewed. Cognitive impairment without dementia was defined as impairment in at least one domain of the neuropsychological test battery using education adjusted cut-off values of 1.5 SDs below the established normal means on individual tests. Failure in at least half of the tests in a domain constituted failure in that domain. CIND mild was diagnosed when $\leq 2$ domains were impaired and CIND moderate as impairment of $>2$ domains.

A diagnosis of dementia was made according to the DSM-IV criteria. The aetiological diagnosis of Alzheimer's disease was made using the criteria for vascular dementia of the National Institute of Neurological and Communicative Disorders and Stroke-Alzheimer's Disease and Related Disorders Association (NINCDS-ADRDA) and the National Institute of Neurological Disorders and Stroke-Association Internationale pour la Recherché et l' Enseignement en Neurosciences (NINDSAIREN). ${ }^{30}$ Dementia severity was assessed using the Clinical Dementia Rating Scale.

\section{Associated factor assessment}

The associated risk factors (age, gender, education, hypertension, diabetes, hyperlipidaemia, smoking, alcohol consumption, low socioeconomic status, body mass index) were assessed under standardised conditions. Systolic and diastolic blood pressures were measured using a digital automatic blood pressure monitor (OMRON-HEM 7203, Japan) after subject rested for $5 \mathrm{~min}$. Blood pressure was measured twice, $5 \mathrm{~min}$ apart. The mean of the two readings was considered the relevant blood pressure. Hypertension was defined as systolic blood pressure $\geq 140 \mathrm{~mm} \mathrm{Hg}$ and/or diastolic blood pressure $\geq 90 \mathrm{~mm} \mathrm{Hg}$ or use of antihypertensive medications. Diabetes was defined as receiving treatment or glycated haemoglobin $\geq 6.5 \%$. Hyperlipidaemia was defined as those on medications or total cholesterol levels $\geq 4.14 \mathrm{mmol} / \mathrm{l}$ following the National Cholesterol Education Programme Adult Treatment Panel III guidelines. Education was categorised into no formal education and formal education ( $\geq$ primary 1 ). Smoking was categorised as non-smokers and smokers (past and current smokers). Alcohol consumption was divided into non-drinkers and drinkers. A low socioeconomic status was defined as a monthly income <SGD2000. Body mass index was calculated as weight $(\mathrm{kg})$ divided by the square of height $(\mathrm{m})$.

\section{Statistical analysis}

Statistical analysis was performed using standard statistical software (Statistical Package for Social Science, SPSS V.19, SPSS Inc, USA). To examine differences in associated factors between screen positive subjects who participated in phase II and those who did not, we used the Student's t test for normally distributed continuous variables (body mass index), the Mann-Whitney $\mathrm{U}$ test for skewed distributed continuous variables (age) and the $\chi^{2}$ test for categorical variables. Crude and age adjusted prevalence of cognitive impairment and dementia were computed for the whole cohort and within the strata of age and gender. Age standardised rates were computed using world standard population $^{31} 32$ and CIs were calculated assuming that the prevalence counts were Poisson distributed. To determine the relationship between associated factors and cognitive impairment/dementia, multiple logistic regression models were used, and ORs with 95\% CI were computed. In model I, we included age, gender and education, and each associated factor separately. In model II, all the associated factors were included to examine whether they were independently associated with cognition.

In order to include those screen positive subjects who did not participate in phase II, we performed sensitivity analyses by using different approaches. Two simple approaches assumed that all screen positive non-responders were either all cognitively normal (conservative approach) or impaired (liberal approach), respectively. Finally, the third approach used was multiple imputation that was taken to estimate the cognitive impairment status of non-responders based on the data of the responders in phase II. Multiple imputation by chain equations was used to impute the missing values. Analysis of these multiple imputed data consists of extracting the estimate and its SE from each analysis performed on an imputed completed dataset, and combining the multiple estimates and SEs to obtain a single estimate and CI for the age standardised prevalence rates of cognitive impairment and dementia. The linear regression model is used to model missing continuous variables and logistic regression is used to model missing categorical variables. For the multiple imputation analysis, we performed 20 imputations. Finally, we compared our gender specific prevalence estimates with those reported from previous studies.

\section{RESULTS}

Screening and assessment of the Chinese cohort was performed from 12 August 2010 to 10 February 2012. For those $\geq 60$ years, participation rates for the first phase were as follows: $73.6 \% \quad(595 / 808)$ for 60-64 years, $70.8 \%$ (361/510) for 65-69 years, $70.5 \% \quad(335 / 475)$ for $70-74$ years, $55.8 \%$ $(173 / 310)$ for $75-79$ years and $49.0 \%(74 / 151)$ for those aged $\geq 80$ years. Of the 1538 subjects (mean age 68.9 years; range 60-85 years), 612 participants were screen positive. Of these, 300 subjects agreed to participate in phase II. Compared with those who did not participate in phase II $(n=312)$, those who 
Table 1 Baseline characteristics of screen positive participants in phase II compared with non-participants

\begin{tabular}{|c|c|c|c|c|}
\hline \multirow[b]{2}{*}{ Risk factors } & \multirow{2}{*}{$\begin{array}{l}\text { Participants in phase I } \\
\text { Yes }(n=300)\end{array}$} & \multicolumn{2}{|c|}{ Participated in phase II } & \multirow[b]{2}{*}{ p Value } \\
\hline & & $(n=1538)$ & No $(n=312)$ & \\
\hline Age (years) & $68.9(60-85)$ & $69.9(60-85)$ & $71.4(60-85)$ & 0.002 \\
\hline Gender (\% women) & $725(47.1)$ & $156(52.0)$ & $172(55.1)$ & 0.438 \\
\hline No formal education (\% no) & $600(39.0)$ & $144(40.9)$ & $171(55)$ & $<0.001$ \\
\hline Socioeconomic status ( $\%$ low) & $992(64.5)$ & $223(63.4)$ & $242(77.8)$ & $<0.001$ \\
\hline Diabetes $(\%)$ & $333(21.7)$ & $96(27.3)$ & 77 (24.8) & 0.462 \\
\hline Hyperlipidaemia (\%) & 807 (52.5) & $271(77)$ & $147(47.3)$ & $<0.001$ \\
\hline Hypertension (\%) & $1195(77.7)$ & $261(74.1)$ & $258(83)$ & 0.006 \\
\hline Smoking (\% yes) & $448(29.1)$ & $101(28.7)$ & $93(29.9)$ & 0.742 \\
\hline Alcohol drinking (\% yes) & $140(9.1)$ & $27(7.7)$ & $19(6.1)$ & 0.407 \\
\hline Body mass index $\left(\mathrm{kg} / \mathrm{m}^{2}\right)$ & $23.6(3.6)$ & $23.8(3.8)$ & $23.5(3.4)$ & 0.217 \\
\hline
\end{tabular}

${ }^{*} \mathrm{p}$ Value for comparison between subjects who participated in phase II and those who did not.

participated were younger (mean age 69.9 years), more often women, had a higher education and higher socioeconomic status and were less often hypertensive, whereas the proportion of hyperlipidaemia was higher (table 1). After excluding these 312 subjects, a total of 1226 subjects were available for the analysis (926 screen negatives and 300 screen positives). Mean age of these 1226 subjects was 68.2 years (range 60-85 years). Among these subjects, only 59 (4.9\%) were $>80$ years of age.

Table 2 shows the crude and age standardised prevalence of cognitive impairment. Among 1226 subjects, 171 participants were diagnosed with cognitive impairment, of whom 84 (6.9\%) had CIND mild, $80(6.5 \%)$ had CIND moderate and seven $(0.5 \%)$ had dementia. However, due to the small numbers, dementia was combined with CIND moderate for further analyses. The crude prevalence of any cognitive impairment in the population was $13.9 \%$, with an age standardised prevalence rate of $15.2 \%$ (95\% CI 12.8 to 17.6$)$. The age standardised prevalence of CIND mild was 7.2\% (95\% CI 5.6 to 8.8) and CIND moderate/dementia $7.9 \%$ (95\% CI 6.2 to 9.7). Prevalence increased with age, from $5.9 \%$ in those aged $60-64$ years to $31.3 \%$ in those $75-79$ years and $44.1 \%$ in those aged $>80$ years. In the present study, multivariate analyses revealed

Table 2 Prevalence of cognitive impairment and dementia both overall and stratified by age and gender

\begin{tabular}{|c|c|c|c|c|c|c|}
\hline \multirow[b]{2}{*}{ Age } & \multicolumn{2}{|c|}{ All persons } & \multicolumn{2}{|l|}{ Men } & \multicolumn{2}{|c|}{ Women } \\
\hline & No & n (\%) & No & n (\%) & No & $\mathrm{n}(\%)$ \\
\hline \multicolumn{7}{|c|}{ Any cognitive impairment } \\
\hline $60-64$ years & 495 & $29(5.9)$ & 279 & $16(5.7)$ & 216 & $13(6.0)$ \\
\hline $65-69$ years & 275 & $34(12.4)$ & 140 & $10(7.1)$ & 135 & $24(17.8)$ \\
\hline $70-74$ years & 266 & $41(15.4)$ & 152 & $20(13.2)$ & 114 & $21(18.4)$ \\
\hline $75-79$ years & 131 & $41(31.3)$ & 76 & $17(22.4)$ & 55 & $24(43.6)$ \\
\hline$\geq 80$ years & 59 & $26(44.1)$ & 26 & $6(23.1)$ & 33 & $20(60.6)$ \\
\hline Crude & 1226 & $171(13.9)$ & 673 & $69(10.3)$ & 553 & $102(18.4)$ \\
\hline Adjusted & & $15.2(12.8-17.6)^{*}$ & & $10.6(7.8-13.3)^{*}$ & & $19.9(15.9-23.8)^{*}$ \\
\hline \multicolumn{7}{|l|}{ CIND mild } \\
\hline $60-64$ years & 495 & $22(4.4)$ & 279 & $13(4.7)$ & 216 & $9(4.2)$ \\
\hline $65-69$ years & 275 & $19(6.9)$ & 140 & $7(5.0)$ & 135 & $12(8.9)$ \\
\hline $70-74$ years & 266 & $20(7.5)$ & 152 & $10(6.6)$ & 114 & $10(8.8)$ \\
\hline $75-79$ years & 131 & $14(10.7)$ & 76 & $9(11.8)$ & 55 & $5(9.1)$ \\
\hline$\geq 80$ years & 59 & $9(15.3)$ & 26 & $2(7.7)$ & 33 & $7(21.2)$ \\
\hline Crude & 1226 & $84(6.9)$ & 673 & $41(6.1)$ & 553 & $43(7.8)$ \\
\hline Adjusted & & $7.2(5.6-8.8)^{\star}$ & & $6.0(4.1-8.0)^{*}$ & & $8.3(5.8-10.8)^{*}$ \\
\hline \multicolumn{7}{|c|}{ CIND moderate/dementia } \\
\hline $60-64$ years & 495 & $7(1.4)$ & 279 & $3(1.1)$ & 216 & $4(1.9)$ \\
\hline $65-69$ years & 275 & $15(5.5)$ & 140 & $3(2.1)$ & 135 & $12(8.9)$ \\
\hline $70-74$ years & 266 & $21(7.9)$ & 152 & $10(6.6)$ & 114 & $11(9.6)$ \\
\hline $75-79$ years & 131 & $27(20.6)$ & 76 & $8(10.5)$ & 55 & $19(34.5)$ \\
\hline$\geq 80$ years & 59 & $17(28.8)$ & 26 & $4(15.4)$ & 33 & $13(39.4)$ \\
\hline Crude & 1226 & $87(7.1)$ & 673 & $28(4.2)$ & 553 & $59(10.7)$ \\
\hline Adjusted & & $7.9(6.2-9.7)^{\star}$ & & $4.5(2.7-6.4)^{\star}$ & & $11.6(8.5-14.6)^{*}$ \\
\hline
\end{tabular}

${ }^{*}$ Age standardised rates $(95 \% \mathrm{Cl})$ compared with world standard population.

CIND, cognitive impairment no dementia. 
Table 3 Association between baseline risk factors and cognitive impairment, expressed as OR with corresponding $95 \% \mathrm{Cl}$

\begin{tabular}{|c|c|c|c|c|}
\hline \multirow[b]{2}{*}{ Risk factors } & \multicolumn{2}{|l|}{ CIND mild ( $n=84)$} & \multicolumn{2}{|c|}{ CIND moderate/dementia ( $n=87)$} \\
\hline & Model I* & Model IIt & Model I* & Model IIt \\
\hline Age (years) & $1.08(1.04$ to 1.12$)$ & $1.11(1.05$ to 1.18$)$ & 1.18 (1.13 to 1.22$)$ & 1.17 (1.12 to 1.21$)$ \\
\hline Gender (women vs men) & $1.06(0.69$ to 1.63$)$ & $0.90(0.50$ to 1.53$)$ & 2.37 (1.44 to 3.88$)$ & 2.18 (1.16 to 4.12$)$ \\
\hline No formal education & 1.81 (1.16 to 2.84$)$ & 1.93 (1.09 to 3.40$)$ & 1.46 (0.89 to 2.41$)$ & 1.04 (0.58 to 1.86$)$ \\
\hline Low socioeconomic status & 1.13 (0.63 to 2.03 ) & 1.13 (0.61 to 2.07 ) & 2.16 (1.04 to 4.45$)$ & 2.04 (0.96 to 4.31$)$ \\
\hline Diabetes & $1.48(0.92$ to 2.39$)$ & 1.85 (1.11 to 3.05$)$ & 1.97 (1.18 to 3.30$)$ & 2.11 (1.23 to 3.61$)$ \\
\hline Hyperlipidaemia & 2.32 (1.44 to 3.74$)$ & 2.85 (1.70 to 4.77$)$ & 3.08 (1.75 to 5.39$)$ & 3.22 (1.82 to 5.67$)$ \\
\hline Hypertension & 0.66 (0.40 to 1.08$)$ & 0.64 (0.37 to 1.11$)$ & $1.00(0.52$ to 1.93$)$ & 0.91 (0.46 to 1.79 ) \\
\hline Smoking & 1.03 (0.60 to 1.77$)$ & $0.93(0.52$ to 1.65$)$ & 1.52 (0.80 to 2.89$)$ & 1.34 (0.68 to 2.60$)$ \\
\hline Alcohol drinking & 1.30 (0.57 to 2.96$)$ & 1.15 (0.48 to 2.67$)$ & 1.15 (0.43 to 3.09$)$ & 0.99 (0.35 to 2.72 ) \\
\hline Body mass index & 0.98 (0.89 to 1.08$)$ & $0.95(0.80$ to 1.10$)$ & $0.99(0.89$ to 1.10$)$ & 0.98 (0.88 to 1.11$)$ \\
\hline
\end{tabular}

* Model I included age, gender, education and each associated factor separately.

tModel II included age, gender, education, low socioeconomic status, diabetes, hyperlipidaemia, hypertension, smoking, alcohol drinking and body mass index.

CIND, cognitive impairment no dementia.

that age, gender, presence of diabetes and hyperlipidaemia were independently associated with cognitive impairment (table 3).

Finally, we performed a sensitivity analysis for the prevalence of cognitive impairment/dementia by including the screen positive non-responders $(n=312)$ using different approaches (table 4). The two simple approaches revealed a broad range (11.1-31.3\%) for the prevalence of cognitive impairment/ dementia in this Chinese population. However, as these two approaches reflect the two possible extremes, we used a third more realistic approach using multiple imputation to estimate the prevalence. This approach gave an overall prevalence of cognitive impairment/dementia of $24.3 \%$. Trends for cognitive impairment across age and gender were the same as those found when we performed the initial analyses excluding the screen positive non-responders. Figure 2 shows our overall and gender specific prevalence estimates in relation to those reported from previous studies on cognitive impairment. Our prevalence estimates were comparable with those described in other studies.

\section{DISCUSSION}

In this population based study among Singaporean Chinese aged $\geq 60$ years, the prevalence of all cognitive impairment was $15.2 \%$, with CIND mild accounting for $7.2 \%$ and CIND moderate/dementia for $7.9 \%$. The prevalence of cognitive impairment increased from $5.9 \%$ in those aged $60-64$ years to $44.1 \%$ in those aged $\geq 80$ years. The most important associated factors were age, diabetes and hyperlipidaemia.

Some limitations of our study should be noted. Firstly, nearly half of the screen positives declined to take part in phase II. Therefore, our prevalence estimate was probably an underestimation. In order to evaluate the robustness of our prevalence estimates and obtain an indication of the 'true' prevalence, we performed a sensitivity analysis by including the 312 screen positive non-responders using different approaches, including multiple imputation, to estimate their cognitive status in phase II. This approach suggests that the true prevalence may be around 24\% in this Chinese population. Secondly, due to the relatively few cases of dementia, we were not able to study dementia separately. Furthermore, we were unable to examine the associated factors of dementia subtypes due to the small numbers. Thirdly, because of the cross sectional design of the study, it was not possible to make causal inferences with respect to the relationship we found between the associated factors and cognitive impairment. Finally, the small number of subjects and a relatively low participation rate for those $>75$ years of age (in particular $>80$ years of age) limited us in extrapolating our findings to these age groups.

The strengths of the study include the following: subjects in the EDIS study were drawn from a population based study; extensive neuropsychological examination for diagnosing cognitive impairment and dementia; and standardised procedures to collect data on baseline associated factors.

Comparison of our study in terms of the prevalence of cognitive impairment with other Caucasian and Asian studies is challenging due to differences in the selection of study populations, demographic differences between populations (such as age distributions), differences in clinical criteria used and disparity in the definitions of cognitive impairment. A recent systemic review included all of the population based studies from 1984 and 2008 which examined the prevalence of cognitive

Table 4 Sensitivity analysis on the prevalence of cognitive impairment and dementia by including the 312 non-responders at phase II ( $\mathrm{n}=1538$ )

\begin{tabular}{|c|c|c|c|c|}
\hline \multirow[b]{2}{*}{ Approach } & \multirow[b]{2}{*}{ Type of impairment } & \multicolumn{3}{|c|}{ Age standardised rates $(95 \% \mathrm{Cl})$} \\
\hline & & All persons & Men & Women \\
\hline Non-respondents: not cognitively impaired & Any cognitive impairment & $11.1(9.4$ to 12.9$)$ & $8.3(6.2$ to 10.4$)$ & $13.8(11.1$ to 16.5$)$ \\
\hline Non-respondents: cognitively impaired & Any cognitive impairment & $31.3(28.4$ to 34.2$)$ & 25.4 (21.7 to 29.1$)$ & 37.1 (32.7 to 41.5$)$ \\
\hline Multiple imputation & Any cognitive impairment & $24.3(21.6$ to 27.0$)$ & $18.6(15.1$ to 22.1$)$ & $29.8(25.7$ to 34.0$)$ \\
\hline Multiple imputation & CIND mild & $11.4(9.4$ to 13.4$)$ & $10.0(7.2$ to 12.9$)$ & 12.7 (9.6 to 15.7$)$ \\
\hline Multiple imputation & CIND moderate/dementia & 12.9 (10.6 to 15.2$)$ & 8.5 (5.8 to 11.2 ) & 17.2 (13.5 to 20.8 ) \\
\hline
\end{tabular}


Figure 2 Prevalence of cognitive impairment from different studies with subjects aged $60-80$ years. Reference line represents the prevalence of cognitive impairment in our present study.
Plot of Prevalence

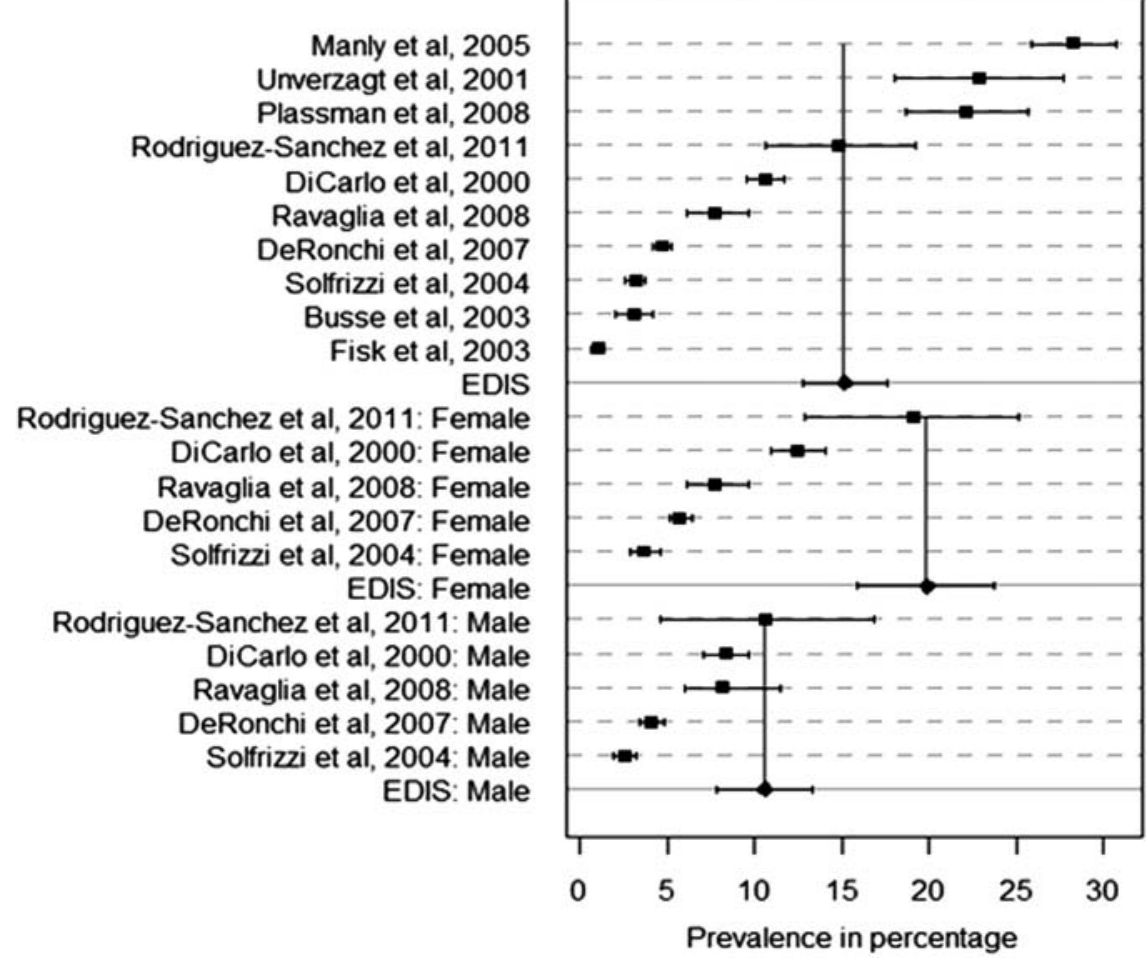

Manly et al, 2005

Unverzagt et al, 2001

Sanchez et al, 2011

DiCarlo et al, 2000

Ravaglia et al, 2008

eRonchi et al, 2007

olfrizzi et al, 2004

Busse et al, 2003

2003

EDIS

Ravaglia et al, 2008: Female

eRonchi et al, 2007: Female

2004: Female

EDIS: Female

Sanchez

DiCarlo et al, 2000: Male

Ravaglia et al, 2008: Male

DeRonchi et al, 2007: Male

EDIS: Male

Prevalence in percentage impairment. ${ }^{33}$ Since the publication of this review, one additional study from Spain has also reported prevalence estimates for cognitive impairment. ${ }^{34}$ Figure 2 summarises the studies from this review, which reported age adjusted prevalence estimates together with our own prevalence estimates. Figure 2 shows that the reported prevalence of cognitive impairment has a wide range, from $1.03 \%$ to $28.3 \%$, and that the prevalence estimate from the EDIS is intermediate. In our study, the prevalence estimate in women (20\%) was higher compared with men (11\%), which is in accordance with findings from previous studies. As this is probably an underestimation, our imputed prevalence of $24.3 \%$ suggests that the true prevalence among Singaporean Chinese may be towards the higher end of this range. Specifically, with respect to Asian studies among subjects who were 65 years and older, a study from Japan reported an overall crude prevalence of $10.8 \% 0^{35}$ whereas another study from Taiwan showed a crude prevalence of $22.2 \% .^{7}$ However, a direct comparison with our study is not possible as they presented only crude estimates and did not use extensive neuropsychological assessment for the diagnosis of cognitive impairment and dementia.

With respect to associated factors for cognitive impairment, we found a large difference in the prevalence of cognitive impairment between genders, with women nearly twice as likely of having cognitive impairment. ${ }^{36} 37$ Apart from gender, we found that age, and those with diabetes and hyperlipidaemia were more likely to have cognitive impairment. These findings are in accordance with findings from previous studies.

In conclusion, this study showed an overall prevalence of $15.2 \%$ for cognitive impairment and dementia in a Chinese population in Singapore, which is in accordance with data from other Caucasian and Asian studies. These data further underline the fact that future research should focus on the preclinical stages of dementia, not only in Caucasian, but equally so in Asian populations. Future studies, including those with a longitudinal design, are required to establish risk factors for the development of these preclinical stages and progression of cognitive impairment to dementia.
Author affiliations
${ }^{1}$ Department of Pharmacology, National University of Singapore, Singapore ${ }^{2}$ Singapore National Eye Centre, Singapore Eye Research Institute, Singapore ${ }^{3}$ Department of Ophthalmology, National University of Singapore, Singapore ${ }^{4}$ Departments of Epidemiology and Ophthalmology, Erasmus Medical Centre, Rotterdam, The Netherlands
${ }^{5}$ Division of Neurology, University Medicine Cluster, National University of Singapore, Singapore
${ }^{6}$ Saw Swee Hock School of Public Health, National University of Singapore, Singapore
${ }^{7}$ Department of Medicine, National University Hospital, Singapore
${ }^{8}$ Department of Diagnostic Imaging, National University Hospital, Singapore
${ }^{9}$ Department of Neurosciences, University of California, La Jolla, California, USA
${ }^{10}$ Department of Bioengineering, National University of Singapore, Singapore
${ }^{11}$ Singapore Institute for Clinical Sciences, The Agency for Science, Technology and Research, Singapore
${ }^{12}$ Clinical Imaging Research Centre, National University of Singapore, Singapore

Contributors SH: acquisition of the data, statistical analysis, interpretation of the data and drafting of the manuscript. MKI: study design and concept, interpretation of the data, study supervision, and drafting and revising the manuscript. MS: acquisition of the data and revising the manuscript. CST: statistical analysis and interpretation of the data. JAC: acquisition of the data and revising the manuscript. YHD: acquisition of the data, study design and concept, and revising the manuscript. LBSL: acquisition of the data and revising the manuscript. EYST: acquisition of the data and revising the manuscript. EHK: study design and concept, and obtained funding. CYLC: acquisition of the data and revising the manuscript. AQ: study design and concept, and revising the manuscript. TYW: study design and concept, obtained funding and revising the manuscript. CL-HC: study design and concept, supervision, obtained funding and revising the manuscript. NV: study design and concept, supervision, obtained funding and revising the manuscript.

Funding This study was funded by the National Medical Research Council, grant No R-184-006-184-511.

\section{Competing interests None.}

Ethics approval The study was approved by the National Healthcare Group Domain-Specific Review Board.

Provenance and peer review Not commissioned; externally peer reviewed. 


\section{REFERENCES}

1 Bremner J, Lee M, Mather M, et al. World population highlights: key findings from PRB'S 2009 world population data sheet, vol 64. Washington, DC, Population Reference Bureau, 2009:16.

2 World Population Prospects: The 2010 Revision (http://www.un.org/esa/population /) (accessed 13 September 2012).

3 Prince M, Jackson J. World Alzheimer's report 2009 executive summary. 2009.

4 Dong MJ, Peng B, Lin XT, et al. The prevalence of dementia in the People's Republic of China: a systematic analysis of 1980-2004 studies. Age Ageing 2007;36:619-24

5 Sahadevan S, Saw SM, Gao W, et al. Ethnic differences in Singapore's dementia prevalence: the stroke, Parkinson's disease, epilepsy, and dementia in Singapore study. J Am Geriatr Soc 2008;56:2061-8.

6 Lim HJ, Lim JP, Anthony P, et al. Prevalence of cognitive impairment amongst Singapore's elderly Chinese: a community-based study using the ECAQ and the IQCODE. Int J Geriatr Psychiatry 2003;18:142-8.

7 Wu MS, Lan TH, Chen CM, et al. Socio-demographic and health-related factors associated with cognitive impairment in the elderly in Taiwan. BMC Public Health 2011;11:22.

8 Foong AW, Saw SM, Loo JL, et al. Rationale and methodology for a population-based study of eye diseases in Malay people: The Singapore Malay eye study (SiMES). Ophthalmic Epidemiol 2007;14:25-35.

9 Lavanya R, Jeganathan VS, Zheng Y, et al. Methodology of the Singapore Indian Chinese Cohort (SICC) eye study: quantifying ethnic variations in the epidemiology of eye diseases in Asians. Ophthalmic Epidemiol 2009;16:325-36.

10 Sahadevan S, Lim PP, Tan NJ, et al. Diagnostic performance of two mental status tests in the older Chinese: influence of education and age on cut-off values. Int J Geriatr Psychiatry 2000;15:234-41.

11 Sahadevan S, Tan NJ, Tan T, et al. Cognitive testing of elderly Chinese people in Singapore: influence of education and age on normative scores. Age Ageing 1997;26:481-6.

12 Hodkinson HM. Evaluation of a mental test score for assessment of mental impairment in the elderly. Age Ageing 1972;1:233-8.

13 Chong MS, Chin JJ, Saw SM, et al. Screening for dementia in the older Chinese with a single question test on progressive forgetfulness. Int J Geriatr Psychiatry 2006;21:442-8.

14 Ong SY, Cheung CY, Li X, et al. Visual impairment, age-related eye diseases, and cognitive function: the Singapore malay eye study. Arch Ophthalmol 2012;12:12.

15 Lawton MP, Brody EM. Assessment of older people: self-maintaining and instrumental activities of daily living. Gerontologist 1969;9:179-86.

16 Mahoney Fl, Barthel DW. Functional evaluation: The Barthel index. Md State Med J $1965 ; 14: 61-5$

17 Yeo D, Gabriel C, Chen C, et al. Pilot validation of a customized neuropsychological battery in elderly Singaporeans. Neurol J South East Asia 1997;123:1-8.

18 Dubois B, Slachevsky A, Litvan I, et al. The FAB: a frontal assessment battery at bedside. Neurology 2000;55:1621-6.
19 Porteus SD. The Maze Test and Clinical Psychology. Palo Alto, CA: Pacific Books, 1959.

20 Wechsler D. Wechsler Memory Scale—revised, 3rd Edn. San Antonio, TX: Jovanovich, 1997.

21 Lewis RF, Rennick PM. Manual for the Repeatable Cognitive Perceptual-Motor Battery. Clinton Township, MI: Axon, 1979.

22 Mack WJ, Freed DM, Williams BW, et al. Boston Naming Test: shortened versions for use in Alzheimer's disease. J Gerontol 1992;47:154-8.

23 Isaacs B, Kennie AT. The set test as an aid to the detection of dementia in old people. Br J Psychiatry 1973;123:467-70.

24 Smith A. Symbol Digit Modalities Test. Los Angeles, CA, The American Psychiatric Pub, 1973.

25 Diller L, Ben-Yishay Y, Gerstman LJ. Studies in cognition and rehabilitation in hemiplegia. New York: New York University Medical Center Institute of Rehabilitation Medicine, 1974.

26 Sunderland T, Hill JL, Mellow AM, et al. Clock drawing in Alzheimer's disease. A novel measure of dementia severity. J Am Geriatr Soc 1989;7:725-9.

27 Wechsler D. Wechsler Adult Intelligence Scale_-Revised. San Antonio, TX: Harcourt Brace Jovanovich, 1981.

28 Yesavage JA, Brink TL, Rose TL, et al. Development and validation of a geriatric depression screening scale: a preliminary report. J Psychiatr Res 1982;7:37-49.

29 Cummings JL. The neuropsychiatric inventory: assessing psychopathology in dementia patients. Neurology 1997;8:S10-16.

30 McKhann GM, Knopman DS, Chertkow $\mathrm{H}$, et al. The diagnosis of dementia due to Alzheimer's disease: recommendations from the National Institute on Aging-Alzheimer's Association workgroups on diagnostic guidelines for Alzheimer's disease. Alzheimer Demen: J Alzheimer Assoc 2011;7:263-9.

31 Segi M. Cancer Mortality for Selected Sites in 24 Countries (1950-57). Sendai, Japan: Department of Public Health, Tohoku University of Medicine, 1960.

32 Doll R, Payne P, Waterhouse JAH. Cancer Incidence in Five continents, vol 1, Geneva: Union Internationale Contre le Cancer, 1966.

33 Ward A, Arrighi HM, Michels S, et al. Mild cognitive impairment: disparity of incidence and prevalence estimates. Alzheimers Dement 2012;8:14-21.

34 Rodriguez-Sanchez E, Mora-Simon S, Patino-Alonso MC, et al. Prevalence of cognitive impairment in individuals aged over 65 in an urban area: DERIVA study. BMC Neurol 2011;1:147.

35 Kumamoto T, Sannomiya K, Ueyama $\mathrm{H}$, et al. Neurological abnormalities in cognitively impaired but not demented elderly. Acta Neurol Scand 2000;02:292-8.

36 Graham JE, Rockwood K, Beattie BL, et al. Prevalence and severity of cognitive impairment with and without dementia in an elderly population. Lancet 1997:49:1793-6.

37 Ebly EM, Hogan DB, Parhad IM. Cognitive impairment in the nondemented elderly. Results from the Canadian study of health and aging. Arch Neurol 1995;2:612-19. 